\title{
Limits on diffusive shock acceleration in supernova remnants in the presence of cosmic-ray streaming instability and wave dissipation
}

\author{
V. S. Ptuskin and V. N. Zirakashvili
}

\author{
Institute for Terrestrial Magnetism, Ionosphere and Radiowave Propagation of the Russian Academy of Sciences, Troitsk, \\ Moscow Region 142190, Russia
}

Received 23 January 2003 / Accepted 6 February 2003

\begin{abstract}
The instability in the cosmic-ray precursor of a supernova shock moving in interstellar medium is studied. The level of magnetohydrodynamic turbulence in this region determines the maximum energy of particles accelerated by the diffusive shock acceleration mechanism. A high efficiency of cosmic ray acceleration is accepted, and the consideration is not limited by the case of weak turbulence. It is assumed that Kolmogorov-type nonlinear wave interactions together with the ion-neutral collisions restrict the amplitude of the random magnetic field. As a result, the maximum energy of accelerated particles strongly depends on the age of the supernova remnant. The maximum energy can be as high as $\sim 10^{17} \mathrm{ZeV}$ in young supernova remnants and falls to about $\sim 10^{10} \mathrm{Z} \mathrm{eV}$ at the end of the Sedov stage. Thus the standard estimate of maximum particle energy based on the Bohm limit calculated for the interstellar magnetic field strength is not justified in this case. This finding may explain why supernova remnants with age of more than a few thousand years are not prominent sources of very high energy gamma-rays.
\end{abstract}

Key words. ISM: supernova remnants - ISM: cosmic rays - acceleration of particles - shock waves - turbulence gamma rays: theory

\section{Introduction}

Particle acceleration by supernova blast waves is commonly accepted as the main mechanism of acceleration of galactic cosmic rays, see Drury et al. (2001), Malkov \& Drury (2001) for a review. The high density of energetic particles in the shock vicinity where diffusive shock acceleration occurs leads to the modification of gas flow through the action of energetic particle pressure and initiates plasma instabilities produced by the current of energetic particles. The plasma instabilities tend to increase the level of magnetohydrodynamic turbulence which scatters the particles. In the case of strong turbulence $\delta B \sim B_{0}$ $\left(\delta \boldsymbol{B}\right.$ is the amplitude of random magnetic field, $\boldsymbol{B}_{0}$ is the regular magnetic field upstream of the shock), such instabilities may decrease the cosmic ray diffusion coefficient $D$ to the Bohm value $D_{\mathrm{B}}=v r_{\mathrm{g}} / 3$, where $v$ is the particle velocity, $r_{\mathrm{g}}=p c /\left(\mathrm{Ze} \mathrm{B}_{0}\right)$ is the Larmor radius of a particle with momentum $p$ and charge $Z e$. The Bohm value of the diffusion coefficient is commonly used in simulations of cosmic ray acceleration in supernova remnants, e.g. Berezhko et al. (1996).

The dependence of diffusion on energy determines the maximum energy that particles can gain in the process of acceleration. The necessary condition of efficient acceleration at the shock in the free expansion stage is $D(E) \lesssim 0.1 u_{\mathrm{sh}} R_{\mathrm{sh}}$, where $R_{\mathrm{sh}}$ is the radius and $u_{\mathrm{sh}}=\mathrm{d} R / \mathrm{d} t$ is the velocity of spherical

Send offprint requests to: V. S. Ptuskin, e-mail: vptuskin@izmiran.rssi.ru shock. Notice that a typical supernova burst with kinetic energy of ejecta $\mathcal{E}=\mathcal{E}_{51} 10^{51} \mathrm{erg}$ in the interstellar gas with number density $n_{0} \mathrm{~cm}^{-3}$ gives the maximum value of the product $0.1 u_{\mathrm{sh}} R_{\mathrm{sh}} \sim 10^{27}\left(\mathcal{E}_{51} / n_{0}\right)^{0.4} \mathrm{~cm}^{2} \mathrm{~s}^{-1}$ at the end of the free expansion stage of supernova remnant evolution when this product reaches its maximum value. At the same time, the typical value of the cosmic ray diffusion coefficient in the Galaxy is $D_{\text {ISM }}=5.9 \times 10^{28} \beta R_{\mathrm{m}}^{0.3} \mathrm{~cm}^{2} \mathrm{~s}^{-1}$, where $\beta=v / c$ is the dimensionless particle velocity and $R_{\mathrm{m}}=p c / Z$ is the particle magnetic rigidity in units $\mathrm{GV}$, see Jones et al. (2001), and thus the necessary condition of acceleration cannot be fulfilled for relativistic particles unless their diffusion coefficient is anomalously small in the vicinity of the shock. The Bohm value, which is a lower bound of the diffusion coefficient along the magnetic field at $\delta B \lesssim B_{0}$, is equal to $D_{B}=6 \times 10^{21} \beta R_{\mathrm{m}} \mathrm{cm}^{2} / \mathrm{s}$ at $B_{0}=5 \times 10^{-6} \mathrm{G}$. The entire pattern of cosmic ray acceleration at supernova shocks critically depends on the assumption that the energetic particles themselves produce the turbulence needed to provide the anomalously slow diffusion at the site of acceleration. This was perceived at the very beginning of the studies of diffusive shock acceleration (Bell 1978; Lagage \& Cesarsky 1983).

Analyzing the early stage of supernova remnant evolution when the shock velocity is high, $u_{\mathrm{sh}} \sim 10^{4} \mathrm{~km} \mathrm{~s}^{-1}$, Lucek \& Bell (2000), Bell \& Lucek (2001) found that the cosmic ray streaming instability can be so strong that the amplified field $\delta B \gtrsim 10^{-4} \mathrm{G}$ far exceeds the magnetic field in the undisturbed 
medium ahead of the moving shock $B_{0} \sim(2 \div 5) \times 10^{-6} \mathrm{G}$ (see also earlier papers of McKenzie \& Völk 1982; Völk et al. 1984; Falle \& Giddings 1987; Bennett \& Ellison 1995). The maximum particle energy increases accordingly. The cosmic ray acceleration continues at later stages of supernova evolution when the mass of swept-up interstellar gas exceeds the mass of supernova ejecta and the shock velocity goes down according to the Sedov solution. The major portion of galactic cosmic rays is accelerated by the expanding blast wave during this stage. The cosmic-ray streaming instability is less efficient as the shock velocity decreases with time and the nonlinear wave interactions can significantly reduce the level of turbulence at the late Sedov stage (Völk et al. 1988; Fedorenko 1990). This leads to fast diffusion and decreases the maximum energy that can be achieved in a process of diffusive shock acceleration. This effect is aggravated by the wave damping which is due to the ion-neutral collisions if neutral atoms are present in the interstellar gas upstream of the shock (Bell 1978; Drury et al. 1996).

In the present paper, we consider the acceleration of cosmic rays and their streaming instability in a wide range of shock velocities. The level of magnetic field fluctuations is allowed to be arbitrarily large ( $\left.\delta B \gtrless B_{0}\right)$, and the rate of nonlinear wave interactions is assumed to correspond to the Kolmogorov type of nonlinearity. The dissipation of waves by the ion-neutral collisions is also taken into account in the case of a supernova burst in not fully ionized interstellar gas. The task is to find the maximum energy of accelerated particles as a function of the age of the supernova remnant. The problem is enormously complicated by the fact that the processes of wave-particle and wavewave interactions have no universally accepted theoretical description in the case of strong turbulence. So, our consideration is made at the level of approximate estimates and we present not more than a schematic description of the overall picture.

\section{Maximum energy of accelerated particles}

We consider the adiabatic stage of supernova remnant expansion when the major fraction of cosmic rays injected in the interstellar medium is accelerated. The evolution of a shock produced by a supernova outburst in the interstellar medium is described by the Sedov solution, see e.g. Lozinskaya (1992), Ostriker \& McKee (1998). In the case of very efficient acceleration when the cosmic ray pressure inside the supernova envelope governs its expansion, the equations for $R_{\mathrm{sh}}(t)$ and $u_{\mathrm{sh}}(t)$ are:

$$
\begin{aligned}
& R_{\mathrm{sh}}=4.26\left(\frac{\mathcal{E}_{51}}{n_{0}}\right)^{1 / 5}\left(\frac{t}{10^{3} \mathrm{yr}}\right)^{2 / 5} \mathrm{pc}, \\
& u_{\mathrm{sh}}=1.67 \times 10^{3}\left(\frac{\mathcal{E}_{51}}{n_{0}}\right)^{1 / 5}\left(\frac{t}{10^{3} \mathrm{yr}}\right)^{-3 / 5} \mathrm{~km} \mathrm{~s}^{-1} .
\end{aligned}
$$

The self-similar solution (1) is valid under the condition that the mass of swept-up gas significantly exceeds the mass of ejecta $M_{\mathrm{ej}}\left(\sim 1 M_{\odot}\right)$ that is fulfilled at $R_{\mathrm{sh}}>R_{0}=$ $\left(3 M_{\mathrm{ej}} / 4 \pi m_{\mathrm{a}} n_{0}\right)^{1 / 3}=1.9 n_{0}^{-1 / 3} \mathrm{pc}, t>t_{0}=R / u_{0}=190 n_{0}^{-1 / 3} \mathrm{yr}$, where $n_{0}$ is the number density of hydrogen atoms in the interstellar gas, $m_{\mathrm{a}}=1.4 \mathrm{~m}$ is the mean interstellar atom mass per hydrogen nucleus, $m$ is the proton mass and $u_{0} \sim 10^{4} \mathrm{~km} \mathrm{~s}^{-1}$ is the ejecta initial velocity. The preceding evolution of a supernova remnant can be approximated as a free expansion with velocity $u_{0}$. The adiabatic approximation used in the derivation of Eqs. (1) is not valid when the shock velocity sinks below about $u_{\mathrm{sh}}=280 \mathcal{E}_{51}^{0.055} n^{0.111} \mathrm{~km} \mathrm{~s}^{-1}$ and the radiation energy losses become important. The evolution of a supernova shell with intense radiative cooling goes as $R_{\mathrm{sh}} \propto t^{2 / 7}, u_{\mathrm{sh}} \propto t^{-5 / 7}$. We set $\mathcal{E}_{51}=1$ in the subsequent numerical estimates.

In the following, we distinguish $n_{0}$, the mean gas number density that determines the shock wave expansion law, and $n$, the intercloud gas number density. It corresponds to a cloudy structure of interstellar medium with small dense clouds randomly distributed in the diffuse background gas. Two phases of interstellar gas are considered below: a hot ionized component with the temperature of background gas $T=7 \times 10^{5} \mathrm{~K}$, the number densities $n_{0}=10^{-2} \mathrm{~cm}^{-3}, n=3 \times 10^{-3} \mathrm{~cm}^{-3}$, and the magnetic field strength $B_{0}=2 \mu \mathrm{G}$; and a warm partly ionized component with the following parameters: $T=8 \times 10^{3} \mathrm{~K}$, $n_{0}=0.4 \mathrm{~cm}^{-3}, n=0.1 \mathrm{~cm}^{-3}, n_{\mathrm{i}}=0.03 \mathrm{~cm}^{-3}, B_{0}=5 \mu \mathrm{G}$ (where $n_{\mathrm{i}}$ is the number density of ions). We assume that the diffusive shock acceleration of energetic particles should cease to operate after $10^{5}$ years in the hot gas when the shock Mach number falls below $M \sim 3$, and by the time $5 \times 10^{4} \mathrm{yr}$ in the warm gas when the Sedov stage ends.

The acceleration of a fast particle diffusing near the shock front is a version of Fermi type acceleration. The fast particles are scattered on inhomogeneities of the magnetic field frozen into background plasma and they gain energy crossing the shock where the plasma is compressing. In the test particle approximation, the distribution of accelerated cosmic ray particles in momentum for high Mach number shocks has the canonical power-law form $f(p) \propto p^{-4}$ up to some maximum momentum $p_{\max }$. In the case of efficient acceleration, the action of cosmic ray pressure on the shock structure causes nonlinear modification of the shock that changes the shape of particle spectrum making it flatter at relativistic energies, see Berezhko \& Ellison (1999). With allowance made for the nonlinear modification, we assume in the following that the spectrum of energetic particles at the shock is of the form $f(p) \propto p^{-4+a}$ where $0<a<0.5$. The normalization of function $f(p)$ is such that the integral $N=4 \pi \int \mathrm{d} p p^{2} f(p)$ gives the number density of relativistic particles. We assume that the pressure of cosmic rays at the shock $P=(4 \pi / 3) \int \mathrm{d} p p^{3} v f(p)$ is some fraction $\xi_{\text {cr }} \leq 1$ of the upstream momentum flux entering the shock front, so that $P=\xi_{\mathrm{cr}} \rho u_{\mathrm{sh}}^{2}\left(\rho=m_{\mathrm{a}} n_{0}\right)$. The typical value of $\xi_{\mathrm{cr}} \approx 0.5$ and the total compression ratio $\sim 7$ were found in the numerical simulations of strongly modified shocks by Berezhko et al. (1996) under the assumption of efficient dissipation of Alfvénic turbulence in the shock precursor. Thus we accept the following distribution function of cosmic rays at the shock:

$$
\begin{aligned}
& f_{0}(p)=\frac{3 \xi_{\mathrm{cr}} \rho u_{\mathrm{sh}}^{2} H\left(p_{\max }-p\right)}{4 \pi c \varphi\left(p_{\max }\right)} \frac{1}{(m c)^{a} p^{4-a}}, \\
& \varphi\left(p_{\max }\right)=\int_{0}^{p_{\max } / m c} \frac{\mathrm{d} y y^{a}}{\sqrt{1+y^{2}}},
\end{aligned}
$$


where $H(p)$ is the step function. The ultra relativistic asymptotics of $\varphi(p)$ is $\varphi(p) \approx(p / m c)^{a} / a$ and the good approximation at $p / m c \gtrsim 1$ is $\varphi\left(p_{\max }\right) \approx a^{-1}\left(p_{\max } / m c\right)^{a}(1-((1+$ $\left.\left.a)(p / m c)^{a}\right)^{-1}\right)$. The value $a=0.3$ is used in the numerical estimates below.

The spatial distribution of accelerated particles in the plane shock approximation can be roughly presented as

$f_{1}(p, x)=f_{0}(p) \exp \left(u_{\mathrm{sh}} \int_{0}^{x} \frac{\mathrm{d} x_{1}}{D\left(p, x_{1}\right)}\right)$

at $x \leq 0$ (upstream of the shock); and

$f_{2}(p, x)=f_{0}(p)$

at $x \geq 0$ (downstream of the shock).

Here we use the reference frame where the shock is at rest at $x=0$, the gas velocity is parallel to the $x$ axis, and $u_{x}>0$. The steady state one-dimensional solution (3) is valid for $|x| \lesssim$ $\min \left\{R_{\mathrm{sh}}, R_{\mathrm{sh}}(D / u R)^{1 / 2}\right\}$. Equation (3) shows the presence of a cosmic ray precursor of characteristic size $D / u_{\text {sh }}$ ahead of the shock.

The cosmic ray diffusion coefficient is determined by the following approximate equation which can be used for an arbitrary strong turbulence, $\delta B \lessgtr B_{0}$ :

$D=\frac{\left(1+A_{\text {tot }}^{2}\right)^{1 / 2}}{3 A^{2}\left(>k_{\text {res }}\right)} v r_{\mathrm{g}}, k_{\text {res }}=\left(1+A_{\text {tot }}^{2}\right)^{1 / 2} r_{\mathrm{g}}^{-1}$.

We introduce here the dimensionless amplitude of the random field $A_{\text {tot }}^{2}=\delta B^{2} / B_{0}^{2}=\left(4 \pi / B_{0}^{2}\right) \int \mathrm{d} k W(k)$, where $W(k)$ is the energy density of magnetohydrodynamic turbulence. The Larmor radius is defined through the regular field $r_{\mathrm{g}}=p c / Z e B_{0}$. The scattering of particles of Larmor radius $r_{\mathrm{g}}$ is mainly produced by random inhomogeneities with the resonant wave number $k_{\text {res }}$ and the amplitude of resonant waves is characterized by $A^{2}\left(>k_{\text {res }}\right)=4 \pi k_{\text {res }} W\left(k_{\text {res }}\right) / B_{0}^{2}$. We set up the formula (5) with the rounded numerical coefficient as the generalization of the equation for the cosmic ray diffusion coefficient in a weak random field (Berezinskii et al. 1990). In doing the generalization, we distinguish for each energetic particle with momentum $p$ a large-scale magnetic field, that includes the regular magnetic field plus the averaged random magnetic field with $k<k_{\text {res }}(p)$, and a small-scale magnetic field, that includes the random magnetic field with $k>k_{\text {res }}(p)$. The particle moves adiabatically in a large-scale field and experiences scattering by the small-scale field. Note that the choice of numerical coefficient in (5) affects the numerical coefficient in Eq. (7) below.

It is usually assumed that the random field that is necessary for cosmic ray diffusion inside the supernova shell can be produced by the advection of turbulence through the shock front, by the intrinsic instabilities at the shock transition region and by the irregular gas flow downstream of the shock. The turbulence in the upstream region should be due to the cosmic ray streaming instability.

In the case under study, the following steady-state equation determines the energy density $W(k, x)$ of the turbulence amplified by the streaming instability in the cosmic ray precursor upstream of the shock:

$u \frac{\partial W}{\partial x}=2\left(\Gamma_{\mathrm{cr}}-\Gamma_{1}-\Gamma_{\mathrm{nl}}\right) W$

$x \leq 0$.

Here the left hand side of the equation describes the advection of turbulence by gas flow with highly supersonic velocity $u=u_{\mathrm{sh}}$. The terms on the right hand side of the equation describe respectively the wave amplification by cosmic rays, the linear damping of waves in background plasma and the nonlinear wave-wave interactions that limit the amplitude of turbulence. In the strict sense, $\Gamma_{\mathrm{nl}}$ is some integral-differential operator acting on the function $W(k, x)$.

The diffusion current due to the nonuniform distribution of cosmic ray particles upstream of the shock causes the streaming instability and the amplification of magnetohydrodynamic waves with the growth rate

$$
\Gamma_{\mathrm{cr}}(k)=\frac{12 \pi^{2} Z^{2} e^{2} V_{\mathrm{a}}\left(1+A_{\mathrm{tot}}^{2}\right)^{1 / 2}}{c^{2} k}
$$

(the waves with $k_{x}<0$ are amplified). Here $p_{\text {res }}(k)=$ $Z e B_{0}\left(1+A_{\text {tot }}^{2}\right)^{1 / 2} / c k$, and the Alfvén velocity is defined through the regular field $B_{0}$ as $V_{\mathrm{a}}=B_{0} / \sqrt{4 \pi \rho}=$ $1.8\left(B_{0} / 1 \mu \mathrm{G}\right) n^{-1 / 2} \mathrm{~km} \mathrm{~s}^{-1}$. The most abundant proton component of cosmic rays determines the value of $\Gamma_{\mathrm{cr}}$, so we put $Z=1$ in the subsequent calculations. The expression (7) is the generalization of the equation for a weak random field (Berezinskii et al. 1990) to the case of strong random field.

Equations (2), (3), (5), (7) let us estimate the value of growth rate in the upstream region $(x \leq 0)$ as

$$
\begin{aligned}
\Gamma_{\mathrm{cr}}(k) \approx & \frac{C_{\mathrm{cr}}(a) \xi_{\mathrm{cr}} u_{\mathrm{sh}}^{3} k^{1-a}\left(1+A_{\mathrm{tot}}^{2}\right)^{-(1-a) / 2}}{c V_{\mathrm{a}} \varphi\left(p_{\mathrm{max}}\right) r_{\mathrm{g} 0}^{a}} \\
& \times \exp \left(3 u_{\mathrm{sh}} \int_{0}^{x} \mathrm{~d} x_{1} \frac{k A^{2}\left(>k, x_{1}\right)}{v\left(1+A_{\mathrm{tot}}^{2}\right)}\right)
\end{aligned}
$$

at $k \geq k_{\mathrm{res}}\left(p_{\mathrm{max}}\right)$, and $\Gamma_{\mathrm{cr}}=0$ otherwise. Here $r_{\mathrm{g} 0}=m c^{2} /\left(e B_{0}\right)$, $C_{\mathrm{cr}}(a)=\frac{27}{4(5-a)(2-a)}$, so that $C_{\mathrm{cr}}(0.3) \approx 0.845$.

Considering that all waves upstream of the shock are generated by cosmic-ray streaming instability, the particles with maximum momentum $p_{\max }$ are in resonance with the waves that have the minimum wave number $k_{\min }=$ $\left(1+A_{\mathrm{tot}}^{2}\right)^{1 / 2} r_{\mathrm{g}}^{-1}\left(p_{\max }\right)$. Note that $A^{2}\left(>k_{\min }\right)=A_{\mathrm{tot}}^{2}$.

The most important mechanism of linear damping of Alfvénic turbulence in the problem under consideration is wave dissipation due to ion-neutral collisions, see e.g. 
Kulsrud \& Cesarsky (1971). It occurs in not fully ionized gas and is described by the equations

$$
\begin{aligned}
& \Gamma_{1}=\frac{v_{\text {in }}}{2}, k>v_{\text {in }}\left(1+n_{\mathrm{i}} / n_{\mathrm{H}}\right) /\left(\sqrt{1+A_{\mathrm{tot}}^{2}} V_{\mathrm{a}}\right), \\
& \Gamma_{1}=\frac{k^{2}\left(1+A_{\mathrm{tot}}^{2}\right) V_{\mathrm{a}}^{2}}{2 v_{\mathrm{in}}\left(1+n_{\mathrm{i}} / n_{\mathrm{H}}\right)^{2}}, k \ll \frac{v_{\mathrm{in}}\left(1+n_{\mathrm{i}} / n_{\mathrm{H}}\right)}{\sqrt{1+A_{\mathrm{tot}}^{2}} V_{\mathrm{a}}},
\end{aligned}
$$

where $v_{\text {in }}=n_{\mathrm{H}}\left\langle v_{\mathrm{th}} \sigma\right\rangle \approx 8.4 \times 10^{-9}\left(T / 10^{4} \mathrm{~K}\right)^{0.4}\left(n_{\mathrm{H}} / 1 \mathrm{~cm}^{-3}\right) \mathrm{s}^{-1}$ for temperatures $T \sim 10^{2} \mathrm{~K}$ to $10^{5} \mathrm{~K}$ is the frequency of ionneutral collisions with the cross section $\sigma$ averaged over velocity distribution of thermal particles, $n_{\mathrm{H}}$ is the number density of neutral hydrogen. This gives $v_{\text {in }}=7.7 \times 10^{-10} \mathrm{~s}^{-1}$ in warm diffuse gas.

In spite of great progress in the investigation of magnetohydrodynamic turbulence, it is not easy to specify $\Gamma_{\mathrm{nl}}$ in Eq. (6). The incompressible MHD simulations of driven and decaying turbulence by Verma et al. (1996) demonstrated the presence of a Kolmogorov-type energy cascade to large wave numbers that can be qualitatively described by the equation

$$
\Gamma_{\mathrm{nl}} W^{ \pm}(k) \approx-\frac{1}{2} C_{\mathrm{K}}^{-3 / 2} \frac{\partial}{\partial k}\left(k^{2} V_{\mathrm{a}}\left(\frac{k W^{\mp}(k)}{B_{0}^{2} / 4 \pi}\right)^{1 / 2} W^{ \pm}(k)\right),
$$

where $C_{\mathrm{K}} \approx 3.6$ is the so-called "Kolmogorov constant" and \pm signs correspond to Alfvén waves travelling in opposite directions, i.e. with $k_{x}>0$ and $k_{x}<0$ respectively, the total wave energy density is $W(k)=W^{+}(k)+W^{-}(k)$, and the turbulence is assumed to be weak, $k W^{\mp}(k)\left(B_{0}^{2} / 4 \pi\right)^{-1} \ll 1$. The nonlinear cascade of Alfvénic waves is anisotropic. The main part of energy density in this turbulence is concentrated perpendicular to the local magnetic field wave vectors $k_{\perp} \approx k$, while the parallel wave numbers are small: $k_{\|} \sim\left(k W(k)\left(B_{0}^{2} / 4 \pi\right)^{-1}\right)^{1 / 2} k_{\perp}$, see Goldreich \& Sridhar (1995), Cho \& Vishniak (2000).

The theoretical approach based on the kinetic theory of weakly turbulent collisionless plasma gives somewhat different results, see Livshits \& Tsytovich (1970), Lee \& Völk (1973), Kulsrud (1978), Achterberg (1981), Fedorenko et al. (1990), Zirakashvili (2000). In particular, it was shown that the nonlinear interactions of Alfvén waves and thermal particles lead to the nonlinear wave damping which is described by the equation

$\Gamma_{\mathrm{nl}} W^{ \pm}(k)=1.1 \sqrt{\beta_{\mathrm{th}}} k V_{\mathrm{a}}\left(\frac{k W^{\mp}(k)}{B_{0}^{2} / 4 \pi}\right) W^{ \pm}(k)$

in the case of high $\beta_{\text {th }}=v_{T}^{2} / V_{\mathrm{a}}^{2}>1$ plasma (Zirakashvili 2000) and by the same within an order of magnitude of the expression but without the factor $\sqrt{\beta_{\text {th }}}$ in the case of low $\beta_{\text {th }} \ll 1$ (Livshits \& Tsytovich 1970; Zirakashvili 2000). Equation (11) was obtained for the case of weak turbulence and under the assumption that wave distribution is not strongly anisotropic in $k$-space. A rate similar to (11) was derived for the wave-wave interactions in magnetohydrodynamics based on the theory of perturbations at small wave amplitude (Chin \& Wentzel 1972; Akhiezer et al. 1975; Skilling 1975).

The observations show that the turbulence in the solar wind plasma is of Kolmogorov type, that evidently favors Eq. (10) over Eq. (11), and contains Alfvén waves moving both along the magnetic field and in perpendicular directions (Saur \& Bieber 1999). The data on interstellar turbulence are also consistent with the assumption that a single Kolmogorov spectrum extends from scales $10^{8}$ to $10^{20} \mathrm{~cm}$ (Armstrong et al. 1995).

It is clear from expressions (10) and (11) that Alfven waves with opposite signs of $k_{x}$ are needed for nonlinear interactions. (The only exception is the one-dimensional case when the interactions are allowed for the waves propagating in one direction along the external magnetic field, see Lee \& Völk 1973; Kulsrud 1978; Achterberg 1981 and Zirakashvili 2000 for corresponding equations and discussion.) The cosmic-ray streaming upstream of the shock amplifies waves $W^{-}$with $k_{x}<0$ that propagate in the direction of cosmic ray diffusion flux, and damps the waves $W^{+}$with $k_{x}>0$. So, one needs an additional mechanism to maintain the waves in both directions and to assure their efficient nonlinear interactions. This is provided by the scattering of waves on the fluctuations of gas density. Considering the case of the most strong wave interactions, we assume in the following that the isotropization of waves is fast and the waves moving in both directions along the external magnetic field are present. A similar approach is used in the studies of Alfvén waves in the solar wind turbulence, see Goldstein et al. (1995), Hu et al. (1999).

The Kolmogorov-type nonlinearity is assumed in the consideration that follows. The simplified expression

$\Gamma_{\mathrm{nl}}=\left(2 C_{\mathrm{K}}\right)^{-3 / 2} V_{\mathrm{a}} k A(>k) \approx 0.05 V_{\mathrm{a}} k A(>k)$,

at $C_{\mathrm{K}}=3.6$ is accepted in our calculations. The order of magnitude estimate (12) is based on Eq. (10) and is valid for an arbitrary strong turbulence.

It is worth noting that the earlier consideration of cosmic ray streaming instability in supernova remnants by Lagage \& Cesarsky (1983), Völk et al. (1988) and Fedorenko (1990) was made in the limit of $\delta B<B_{0}$ and with the use of an equation similar to (11) that is very different from our present approach.

Let us consider separately the cases of nonlinear and linear dissipation of waves.

A. Cosmic-ray streaming instability with Kolmogorov-type nonlinearity.

The linear damping is ignored in this case. The wave density is determined by Eq. (6) at $\Gamma_{1}=0$ and with $\Gamma_{\mathrm{nl}}$ given by Eq. (12). The exponential distribution of cosmic rays (3) with a characteristic scale $D / u_{\mathrm{sh}}$ determines the spatial distribution of waves in the upstream region that gives the estimate of the first term of Eq. (6) at the shock $u \partial W / \partial x \approx u^{2} W / D$ in the case of efficient wave amplification. Now using Eqs. (5), (8) and (12), one can find from (6) the following approximate equation that determines the amplitude of the random field at the shock:

$$
\begin{aligned}
& \frac{3 u_{\mathrm{sh}}^{2} A^{2}(>k)}{2 v\left(1+A_{\mathrm{tot}}^{2}\right)}+\left(2 C_{\mathrm{K}}\right)^{-3 / 2} V_{\mathrm{a}} A(>k) \\
& =\frac{C_{\mathrm{cr}}(a) \xi_{\mathrm{cr}} u_{\mathrm{sh}}^{3}}{c V_{\mathrm{a}} \varphi\left(p_{\max }\right)\left(k r_{\mathrm{g} 0}\right)^{a}\left(1+A_{\mathrm{tot}}^{2}\right)^{(1-a) / 2}}
\end{aligned}
$$


Equation (13) allows us to find the following equation for $A_{\text {tot }}$ :

$$
\begin{aligned}
& \frac{3 u_{\mathrm{sh}}^{2} A_{\text {tot }}^{2}}{2 v\left(1+A_{\mathrm{tot}}^{2}\right)}+\left(2 C_{\mathrm{K}}\right)^{-3 / 2} V_{\mathrm{a}} A_{\mathrm{tot}} \\
& =\frac{C_{\mathrm{cr}}(a) \xi_{\mathrm{cr}} u_{\mathrm{sh}}^{3}}{c V_{\mathrm{a}} \varphi\left(p_{\max }\right)\left(p_{\max } / m c\right)^{-a}\left(1+A_{\mathrm{tot}}^{2}\right)^{1 / 2}} .
\end{aligned}
$$

As was pointed out in the Introduction, the efficient particle acceleration at the shock necessitates a sufficiently small diffusion coefficient. The maximum momentum of accelerated particles at the Sedov stage of SNR evolution is approximately determined by the condition $D\left(p_{\max }\right)=\varkappa u_{\mathrm{sh}} R_{\mathrm{sh}}$, where $\varkappa \approx 0.04$, see e.g. Berezhko et al. (1996). The particles with a larger diffusion coefficient are not accelerated and are not confined in the remnant. The last condition and Eq. (5) give

$$
\frac{p_{\mathrm{max}}}{m c}=\frac{3 \varkappa A_{\mathrm{tot}}^{2} u_{\mathrm{sh}} R_{\mathrm{sh}}}{\left(1+A_{\mathrm{tot}}^{2}\right)^{1 / 2} v r_{\mathrm{g} 0}} .
$$

As the most abundant species, protons mainly drive the instability. Other particles experience resonant scattering on produced turbulence and undergo diffusive shock acceleration. As a result, the ions with charge $Z$ have the same shape of spectra as protons but written as function of $p / Z$ instead of $p$. This is of course valid for the maximum momentum attained at the shock and $p_{\text {max }} / Z$ should appear in Eq. (15) instead of $p_{\text {max }}$ if $Z \neq 1$ (as before, $m$ is the proton mass, $r_{\mathrm{g} 0}$ is the Larmor radius of a proton with momentum $m c$ in Eq. (15)). The very high energy electrons are accelerated with the same rate as protons at the same magnetic rigidity. However, the maximum energy of electrons is smaller than for protons because of heavy energy losses on synchrotron radiation and inverse Compton scattering, see analysis of these processes by Gaisser et al. (1998), Reynolds (1998).

Equations (14), (15) together with Eqs. (1) determine the maximum momentum of accelerated particles (protons) during the Sedov stage of SNR evolution in an implicit form. The results of numerical solution of these equation for $p_{\max }$ as a function of shock velocity $u_{\mathrm{sh}}$ are presented in Figs. 1 and 2 for the cases of supernova remnant expansion in hot and warm interstellar gas respectively. The values of $\xi_{\mathrm{cr}}=0.5, a=0.3$, and $\mathcal{E}_{51}=1$ were accepted in these calculations. For comparison, the solution without wave damping, $\Gamma_{1}=0, \Gamma_{\mathrm{nl}}=0$, is presented. The Bohm limit for maximum momentum calculated at the interstellar magnetic field strength $B_{0}$ is also shown in Figs. 1 and 2. The Bohm limit represents the standard estimate of maximum momentum of accelerated particles that is commonly accepted in the investigations of cosmic ray acceleration in supernova remnants. Our consideration shows that this "standard" estimate of $p_{\max }$ might be completely unsuitable. It does not work in young supernova remnants at the age $t \lesssim 3 \times 10^{3}$ yr when cosmic ray stream instability generates a strong random magnetic field $\delta B>B_{0}$ that reduces particle diffusion (in agreement with Bell $\&$ Lucek 2001 results). It also does not work in old supernova remnants at $t \gtrsim 3 \times 10^{3} \mathrm{yr}$ when strong Kolmogorov type nonlinear interactions of waves damp the turbulence generated by instability and thus increases particle diffusion upstream of the shock.
It is useful to give simple analytic expressions for $p_{\max }$ in the limits of high and low values of shock velocity $u_{\mathrm{sh}}$.

It is easy to check that if the shock velocity is high enough so that

$u_{\mathrm{sh}} \gg \frac{4 a C_{\mathrm{cr}}(a) \xi_{\mathrm{cr}} c}{9\left(2 C_{\mathrm{K}}\right)^{3 / 2}}, u_{\mathrm{sh}} \gg \frac{3 V_{\mathrm{a}}}{2 a C_{\mathrm{cr}}(a) \xi_{\mathrm{cr}}}$

then the first term in the left-hand side of Eq. (14) dominates over the second term, and the wave amplitude is large, $A_{t} \gg 1$. Thus the advection of waves is more important than the nonlinear wave interactions and the turbulence is strong when (16) is fulfilled. The inequalities (16) are satisfied at $u_{\mathrm{sh}} \gg 8.3 \times 10^{7} \mathrm{~cm} \mathrm{~s}^{-1}, u_{\mathrm{sh}} \gg 7.9 \times 10^{7} \mathrm{~cm} \mathrm{~s}^{-1}$ in hot gas, and $u_{\mathrm{sh}} \gg 8.3 \times 10^{7} \mathrm{~cm} \mathrm{~s}^{-1}, u_{\mathrm{sh}} \gg 3.4 \times 10^{7} \mathrm{~cm} \mathrm{~s}^{-1}$ in warm gas at $a=0.3, \xi_{\text {cr }}=0.5$. Equations (14), (15) now give

$$
\begin{aligned}
\frac{p_{\mathrm{max}}}{m c} & \approx \frac{2 \varkappa a C_{\mathrm{cr}}(a) \xi_{\mathrm{cr}} u_{\mathrm{sh}}^{2} R_{\mathrm{sh}}}{r_{\mathrm{g} 0} V_{\mathrm{a}} c} \\
& \approx 2.2 \times 10^{5}\left(\xi_{\mathrm{cr}} / 0.5\right) \mathcal{E}_{51}^{3 / 5} n_{0}^{-3 / 5} n^{1 / 2} t_{\mathrm{Kyr}}^{-4 / 5}
\end{aligned}
$$

Here we set $\chi=0.04, a=0.3, C_{\mathrm{K}}=3.6$, use Eqs. (1) for $u_{\mathrm{sh}}(t)$ and $R_{\mathrm{sh}}(t)$, and approximate $\varphi\left(p_{\max }\right) \approx\left(p_{\max } / m c\right)^{a} / a$, $v=c ; t_{\mathrm{Kyr}}$ is the supernova remnant age in units of $10^{3} \mathrm{yr}$. Equation (17) estimates $p_{\max } / m c \approx 2 \times 10^{5} t_{\mathrm{Kyr}}^{-4 / 5}$ in hot gas, and $p_{\max } / m c \approx 1 \times 10^{5} t_{\mathrm{Kyr}}^{-4 / 5}$ in warm gas at $t_{\mathrm{Kyr}} \ll 3, \mathcal{E}_{51}=1$, $\xi_{\text {cr }}=0.5$. The cosmic ray diffusion coefficient in the upstream region depends on rigidity as $D \propto r_{\mathrm{g}}^{1-a}$ at $p \lesssim p_{\max }$.

In the opposite limit of low shock velocity

$$
\begin{aligned}
& u_{\mathrm{sh}} \ll\left(\frac{4 V_{\mathrm{a}}^{3} c^{2}}{\pi a C_{\mathrm{cr}}(a)\left(2 C_{\mathrm{K}}\right)^{3} \xi_{\mathrm{cr}}}\right)^{1 / 5}, \\
& u_{\mathrm{sh}} \ll\left(\frac{V_{\mathrm{a}}^{2} c}{a C_{\mathrm{cr}}(a)\left(2 C_{\mathrm{K}}\right)^{3 / 2} \xi_{\mathrm{cr}}}\right)^{1 / 3},
\end{aligned}
$$

the second term in the left-hand side of Eq. (14) dominates over the first term and the wave amplitude is small, $A_{\mathrm{t}} \ll 1$. The non-linear wave interactions are more important than advection in this case. The inequalities (18) are satisfied at $u_{\mathrm{sh}} \ll$ $7.4 \times 10^{7} \mathrm{~cm} \mathrm{~s}^{-1}, u_{\mathrm{sh}} \ll 8.0 \times 10^{7} \mathrm{~cm} \mathrm{~s}^{-1}$ in hot gas, and $u_{\mathrm{sh}} \ll 4.4 \times 10^{7} \mathrm{~cm} \mathrm{~s}^{-1}, u_{\mathrm{sh}} \ll 4.5 \times 10^{7} \mathrm{~cm} \mathrm{~s}^{-1}$ in warm gas. Equations (14), (15) give

$$
\begin{aligned}
\frac{p_{\max }}{m c} & \approx \frac{0.24 \varkappa a^{2} C_{\mathrm{cr}}^{2}(a) C_{\mathrm{K}}^{3} \xi_{\mathrm{cr}}^{2} u_{\mathrm{sh}}^{7} R_{\mathrm{sh}}}{r_{\mathrm{g} 0} V_{\mathrm{a}}^{4} c^{3}} \\
& \approx 4.1 \times 10^{11}\left(\xi_{\mathrm{cr}} / 0.5\right)^{2} \mathcal{E}_{51}^{8 / 5} n_{0}^{-8 / 5} n^{2} B_{0, \mu \mathrm{G}}^{-3} t_{\mathrm{Kyr}}^{-19 / 5}
\end{aligned}
$$

that leads to the estimate $p_{\max } / m c \approx 7 \times 10^{8} t_{\mathrm{Kyr}}^{-19 / 5}$ in hot gas, and $p_{\max } / m c \approx 1 \times 10^{8} t_{\mathrm{Kyr}}^{-19 / 5}$ in warm gas at $t_{\mathrm{Kyr}} \gg 10$. The cosmic ray diffusion coefficient in the upstream region depends on rigidity as $D \propto v r_{\mathrm{g}}^{1-2 a}$ at $p \lesssim p_{\max }$ in this case.

As it is evident from Figs. 1, 2 and Eqs. (17), (19), the value of $p_{\max }$ is rapidly decreasing with the age of supernova remnant $t$. The maximum energy of cosmic rays accelerated by supernova shocks at the early Sedov stage is close to $3 \times 10^{14} \mathrm{eV}$ for protons and decreases to about $10^{10} \mathrm{eV}$ at the end of the Sedov stage for the set of parameters accepted in the present work. In particular, the particle energy is less than $10^{13} \mathrm{eV}$ for 


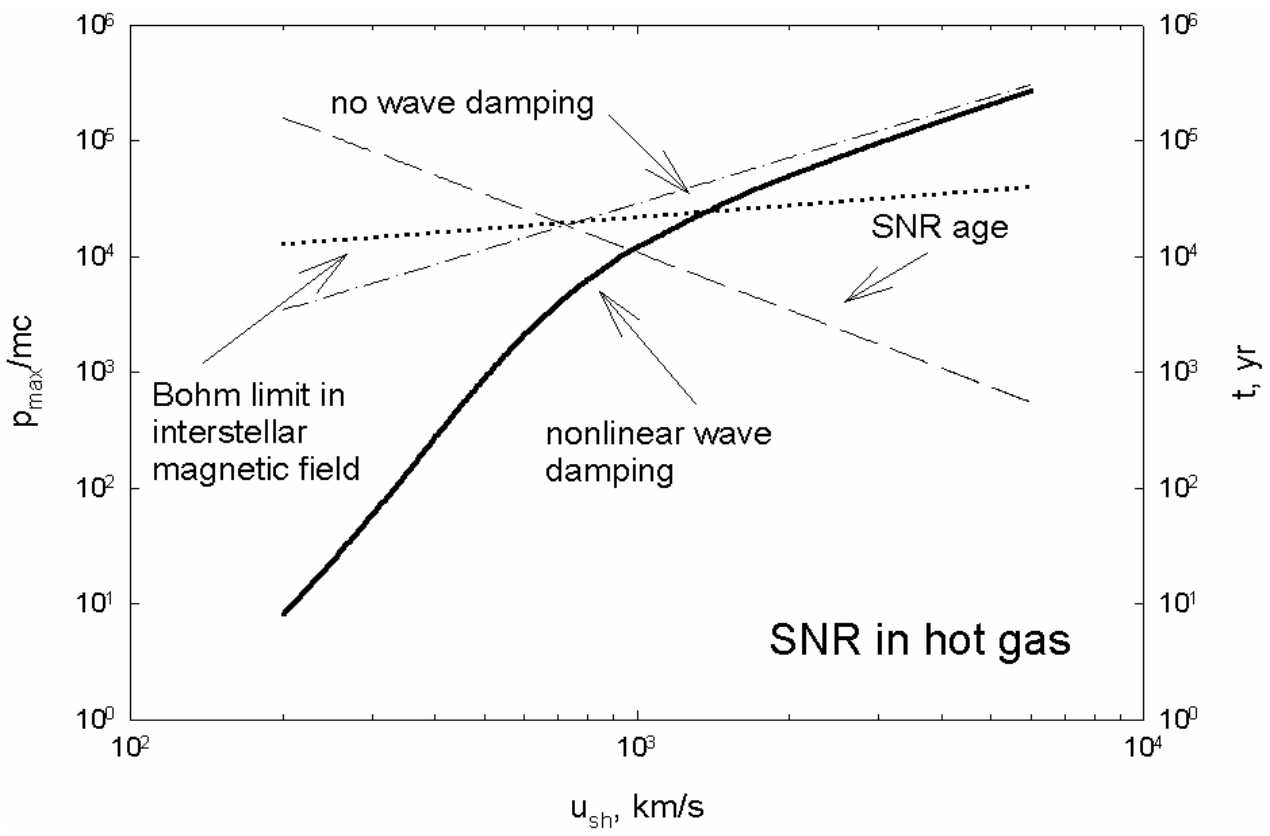

Fig. 1. The maximum momentum of accelerated protons $p_{\max }$ in units of $m c$ as a function of shock velocity $u_{\mathrm{sh}}$ at the Sedov stage of supernova remnant evolution in the hot interstellar gas is shown by the solid line. The kinetic energy of the explosion is $\mathcal{E}=10^{51} \mathrm{erg}$; the efficiency of cosmic ray acceleration $\xi_{\mathrm{cr}}=0.5$. The parameters of interstellar medium are the following: the gas temperature $T=7 \times 10^{5} \mathrm{~K}$, the average gas density $n_{0}=10^{-2} \mathrm{~cm}^{-3}$, the density of diffuse gas $n=3 \times 10^{-3} \mathrm{~cm}^{-3}$, and the interstellar magnetic field strength $B_{0}=2 \mu \mathrm{G}$. The dashed line presents the age of a supernova remnant $t$ (ploted on the right ordinate) as a function of shock velocity. Also shown: dotted line - the Bohm limit on maximum particle momentum in the field $B_{0}$; dash-dot line - the maximum particle momentum when the wave dissipation is not taken into account.

$t \gtrsim 5 \times 10^{3} \mathrm{yr}$ and this may explain the absence of a $\mathrm{TeV}$ gamma-ray signal from many supernova remnants (Buckley et al. 1998) where gamma-rays could be produced through $\pi^{0}$ decays if sufficiently energetic protons and nuclei were present. The commonly accepted estimate of maximum particle energy based on the Bohm diffusion limit enormously over-estimates $p_{\text {max }}$ for these objects if the level of self-generated turbulence is limited by the strong Kolmogorov-type nonlinearity (12).

B. Cosmic-ray streaming instability in warm gas with collisional wave dissipation.

The cosmic ray acceleration in supernova remnants in the presence of wave dissipation by ion-neutral collisions was investigated by Drury et al. (1996). Different aspects of particle acceleration by supernova shocks moving in partly ionized interstellar gas were also considered by Boulares \& Cox (1988), Bykov et al. (2000). It was assumed that the turbulence generated by cosmic ray streaming instability is weak. Here we do not restrict our consideration to the case of weak turbulence. We ignore the nonlinear wave dissipation in this section and consider the collisional dissipation in partly ionized warm gas. The wave density $W(k)$ is determined now from Eq. (6) where the term $\Gamma_{\mathrm{nl}}$ is omitted and the term $\Gamma_{1}$ is given by Eq. (9). So, similar to the case $A$ and associated Eqs. (13), (14), we have the following equation for the amplitude of random magnetic field at the shock:

$\frac{3 u_{\mathrm{sh}}^{2} A^{2}(>k) k}{2 v\left(1+A_{\mathrm{tot}}^{2}\right)}=\frac{C_{\mathrm{cr}}(a) \xi_{\mathrm{cr}} u_{\mathrm{sh}}^{3} k}{c V_{\mathrm{a}} \varphi\left(p_{\max }\right)\left(k r_{\mathrm{g} 0}\right)^{a}\left(1+A_{\mathrm{tot}}^{2}\right)^{(1-a) / 2}}-\frac{v_{\mathrm{in}}}{2}$

at $k \geq k_{*}, k_{*}=v_{\text {in }} /\left(\sqrt{1+A_{\text {tot }}^{2}} V_{\text {a }}\right)$.
Now, using Eqs. (5), (15), (20) and relations $A^{2}\left(>k_{\min }\right)=$ $A_{\text {tot }}^{2}, k_{\min }=\sqrt{1+A_{\text {tot }}^{2}} / r_{\mathrm{g}, \max }$ we find the following equation for $p_{\max }$ :

$\left(\frac{p_{\max }}{m c}\right)^{1-a} \varphi\left(p_{\max }\right)=\frac{2 C_{\mathrm{cr}}(a) \xi_{\mathrm{cr}} u_{\mathrm{sh}}^{3}}{c V_{\mathrm{a}} r_{\mathrm{g} 0}\left(v_{\mathrm{in}}+\frac{u_{\mathrm{sh}}}{\varkappa R_{\mathrm{sh}}}\right)}$

valid for $p_{\max } \leq p_{*}, p_{*} / m c=\left(1+A_{\mathrm{tot}}^{2}\right) V_{\mathrm{a}}\left(v_{\mathrm{in}} r_{\mathrm{g} 0}\right)^{-1}$. The term $v_{\text {in }}$ should be omitted in Eq. (21) if $p_{\max }>p_{*}$. The dissipation on neutrals is not essential for the development of instability if the last inequality is fulfilled.

The value of maximum particle momentum $p_{\text {max }}$ as a function of the supernova shock velocity $u_{\text {sh }}$ determined according to Eq. (21) is shown in Fig. 2. The approximate expression based on Eq. (21) at $v_{\text {in }}>20 u_{\mathrm{sh}} / R_{\mathrm{sh}}$ is

$$
\begin{aligned}
\frac{p_{\mathrm{max}}}{m c} & \approx \frac{2 a C_{\mathrm{cr}}(a) \xi_{\mathrm{cr}} u_{\mathrm{sh}}^{3}}{c V_{\mathrm{a}} r_{\mathrm{g} 0} v_{\mathrm{in}}} \\
& \approx 3 \times 10^{3}\left(\xi_{\mathrm{cr}} / 0.5\right) \mathcal{E}_{51}^{3 / 5} n_{0}^{-3 / 5} n^{-1 / 2} t_{\mathrm{Kyr}}^{-9 / 5}
\end{aligned}
$$

at $a=0.3$ that gives $\left(p_{\max } / m c\right) \approx 5.7 \times 10^{3}\left(u_{\mathrm{sh}} / 10^{3} \mathrm{~km} \mathrm{~s}^{-1}\right)^{3} \approx$ $1.64 \times 10^{4} t_{\mathrm{Kyr}}^{-9 / 5}$ at $\xi_{\mathrm{cr}}=0.5, \mathcal{E}_{51}=1$. The last approximate formula and Fig. 2 show that the maximum energy of accelerated particles decreases from about $3 \times 10^{14} \mathrm{eV}$ in the early Sedov stage to $3 \times 10^{10} \mathrm{eV}$ at the end of the Sedov stage due to the damping of cosmic-ray generated turbulence by ion-neutral collisions. 


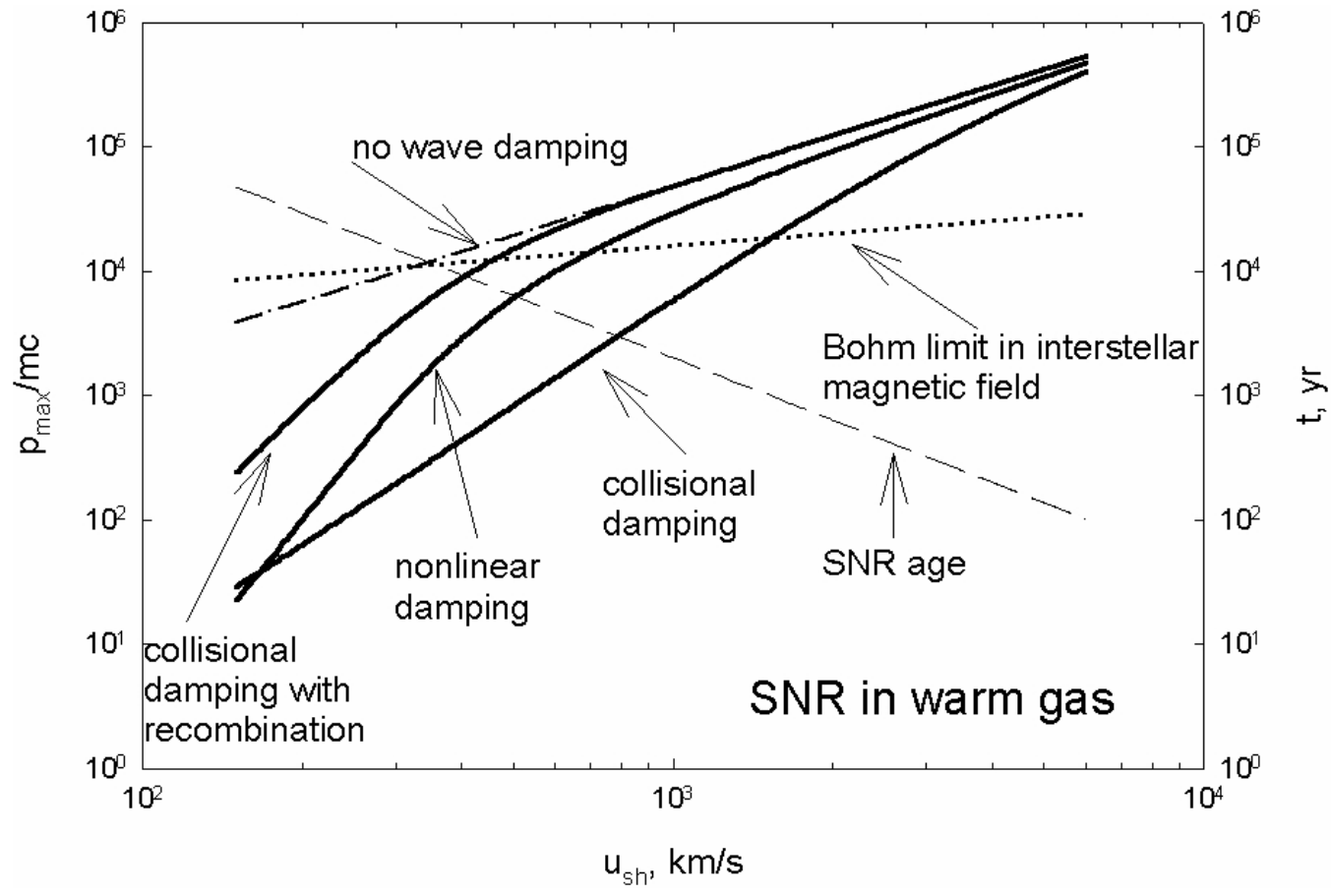

Fig. 2. The same as in Fig. 1 but for the supernova burst in warm interstellar gas $\left(T=8 \times 10^{3} \mathrm{~K}, n_{0}=0.4 \mathrm{~cm}^{-3}, n=0.1 \mathrm{~cm}^{-3}, B_{0}=5 \mu \mathrm{G}\right)$. Three solid lines correspond to three cases of wave dissipation considered separately: nonlinear wave interactions; damping by ion-neutral collisions at given above number density of neutral atoms; damping by ion-neutral collisions when the diffuse neutral gas restores its density after complete ionization by the radiation from supernova burst.

The solution with a smaller value of $p_{\max }$ at given shock velocity $u_{\mathrm{sh}}$ should be chosen when both nonlinear and collisional wave damping are present.

It is known that some envelopes of type Ia supernovae expand in partly ionized interstellar gas (see Lozinskaya 1992), so that the equations obtained above are appropriate in this instance. On the other hand, an explosion of type II supernova leads to the full ionization of surrounding diffuse gas over distances of several tens of parsecs, a condition that holds about $10^{5} n^{-1}$ years after the burst. Therefore, the intercloud neutral gas density is very low shortly after the burst and rises linearly in time because of recombination as

$n_{\mathrm{H}}=C_{\mathrm{r}} n^{2} t$,

at $t \ll\left(C_{\mathrm{r}} n\right)^{-1}$, where $C_{\mathrm{r}}(T)$ is the recombination rate of hydrogen. At a later time $t \sim\left(C_{\mathrm{r}} n\right)^{-1}$ the value of $n_{\mathrm{H}}$ tends to some constant value which is determined by the balance between ionization and recombination. The standard value of recombination rate $C_{\mathrm{r}}=4.1 \times 10^{-13} \mathrm{~cm}^{-3} \mathrm{~s}^{-1}$ at $T \approx 10^{4} \mathrm{~K}$ (Seaton 1959) gives the time-dependent frequency of ion-neutral collisions $v_{\text {in }}(t)=\left\langle v_{\text {th }} \sigma\right\rangle C_{\mathrm{r}} n^{2} t \approx 1 \times 10^{-10} n^{2} t_{\mathrm{Kyr}} \mathrm{s}^{-1}$. One can continue to use Eqs. (20), (21) with the substitution $v_{\text {in }}=v_{\text {in }}(t)$ for the determination of maximum momentum of accelerated particles. Figure 2 illustrates the results. It is evident that the ionization of interstellar atoms by supernova radiation diminishes the collisional wave dissipation.

\section{Supernova explosion in a stellar wind}

Immediately after the supernova burst, the shock propagates not in the interstellar medium but through the wind of a progenitor star. The duration of this phase depends on the wind power and it may cover even the considerable part of the adiabatic (Sedov) stage of supernova remnant evolution if the presupernova star was very massive, more than about 15 solar masses. The massive progenitors of type-II and Ib supernovae undergo a significant mass loss prior to explosion during their red and blue giant phases, and the Wolf-Rayet phase for the most massive stars. The stellar wind modifies the ambient density distribution and creates an expanding shell. The cosmic ray acceleration in the case of shock propagation through the wind was considered by Völk \& Biermann (1988), Berezinsky \& Ptuskin (1989), and Berezhko \& Völk (2000) under the assumption of Bohm diffusion for energetic particles. Bell \& Lucek (2001) included the effect of strong cosmic-ray streaming instability into the consideration of high velocity shocks.

A distinctive property of the problem is the nonhomogeneous distributions of gas density and magnetic field in the stellar wind flow. In particular, the spherically symmetric distribution of gas density in the stellar wind is $n_{\mathrm{w}}=$ $\dot{M} /\left(4 \pi m_{\mathrm{a}} u_{\mathrm{w}} r^{2}\right)$, where $\dot{M}=10^{-5} \dot{M}_{-5} M_{\odot} \mathrm{yr}^{-1}$ is the mass loss, $u_{\mathrm{w}}=10^{6} u_{\mathrm{w}, 6} \mathrm{~cm} \mathrm{~s}^{-1}$ is the wind velocity, and the normalization and numerical values here and below are given for the typical supernova type II progenitor, the red giant star (see Berezinsky \& Ptuskin 1989). Similar to the interplanetary magnetic field, the stellar wind magnetic field has the shape of a Parker spiral (Parker 1958). The magnetic field has predominately azimuthal structure at distance from the star $r \gg u_{\mathrm{w}} /(\Omega \sin \theta) \approx 5 \times 10^{12} / \sin \theta \mathrm{cm}$ where its value is $B_{0}=$ $B_{*} r_{*}^{2} \Omega \sin \theta /\left(u_{\mathrm{w}} r\right)$. Here $B_{*}=10 \mathrm{G}$ is the surface magnetic field strength at star radius $r_{*}=2 \times 10^{13} \mathrm{~cm}, \Omega=2 \times 10^{-7} \mathrm{~s}^{-1}$ is the 
angular velocity of star rotation, and $\theta$ is the polar angle. Hence $B_{0}(r) r \approx 8 \times 10^{14} \sin \theta \mathrm{G} \times \mathrm{cm}$ at $r \gg 5 \times 10^{12} \mathrm{~cm}$. It is worth noting that the value of Alfven velocity $V_{\mathrm{a}}=B_{0}(r) r \sqrt{u_{\mathrm{w}} / \dot{M}}$ does not depend on distance $r$ in this case.

The shock propagation in a stellar wind obeys the following equations (e.g. Lozinskaya 1992):

$$
\begin{aligned}
& R_{\mathrm{sh}}=8.77\left(\frac{u_{\mathrm{w}, 6} \mathcal{E}_{51}}{\dot{M}_{-5}}\right)^{1 / 3} t_{\mathrm{Kyr}}^{2 / 3} \mathrm{pc} \\
& u_{\mathrm{sh}}=5.72 \times 10^{3}\left(\frac{u_{\mathrm{w}, 6} \mathcal{E}_{51}}{\dot{M}_{-5}}\right)^{1 / 3} t_{\mathrm{Kyr}}^{-1 / 3} \mathrm{~km} \mathrm{~s}^{-1}
\end{aligned}
$$

that substitute now Eqs. (1). Expressions (24) are valid at $u_{\mathrm{sh}} \gg u_{\mathrm{w}}$, and under the conditions $R_{\mathrm{sh}}>R_{0}=M_{\mathrm{ej}} u_{\mathrm{w}} / \dot{M} \approx$ $1\left(M_{\mathrm{ej}} / M_{\odot}\right) \mathrm{pc}, t>t_{0} \approx 40\left(M_{\mathrm{ej}} / M_{\odot}\right)^{3 / 2} \mathrm{yr}$ when the mass of swept-up gas is relatively large.

Equations (14), (15) can be used for the estimates of maximum particle momentum when the instability is balanced by the wave advection and nonlinear wave dissipation of Kolmogorov type. The asymptotic expression for $p_{\max }(t)$ at high shock velocity subject to conditions (16) is the following (compare with Eq. (17)):

$$
\begin{aligned}
\frac{p_{\max }}{m c} & \approx \frac{2 \varkappa a C_{\mathrm{cr}}(a) \xi_{\mathrm{cr}} e u_{\mathrm{sh}}^{2} \dot{M}^{1 / 2}}{m c^{3} u_{\mathrm{w}}^{1 / 2}} \\
& \approx 8.9 \times 10^{5}\left(\xi_{\mathrm{cr}} / 0.5\right) \mathcal{E}_{51}^{2 / 3}\left(\frac{u_{\mathrm{w}, 6}}{\dot{M}_{-5}}\right)^{1 / 6} t_{\mathrm{Kyr}}^{-2 / 3} .
\end{aligned}
$$

The asymptotic expression at low shock velocity subject to conditions (18) is

$$
\begin{aligned}
\frac{p_{\max }}{m c} \approx & \frac{0.24 \varkappa a^{2} C_{\mathrm{cr}}^{2}(a) C_{\mathrm{K}}^{3} \xi_{\mathrm{cr}}^{2} \dot{M}^{1 / 2} u_{\mathrm{sh}}^{7}}{V_{\mathrm{a}}^{3} u_{\mathrm{w}}^{1 / 2} c^{5}} \approx 4.6 \times 10^{6} \\
& \times\left(\frac{\xi_{\mathrm{cr}}}{0.5}\right)^{2} \mathcal{E}_{51}^{7 / 3}\left(\frac{u_{\mathrm{w}, 6}}{\dot{M}_{-5}}\right)^{1 / 3}\left(\frac{B_{*} r_{*}}{8 \times 10^{14}}\right)^{3} t_{\mathrm{Kyr}}^{-7 / 3},
\end{aligned}
$$

at $\theta \approx \pi / 2$ (compare with Eq. (19)).

In actuality the regime (26) can hardly be achieved since the size of the wind of a red giant star usually does not exceed $10 \mathrm{pc}$ and the supernova shock breaks out of the wind cavity at $t \lesssim 1 \mathrm{Kyr}$ when the shock velocity is still high. Also, one has to keep in mind that the wind parameters significantly change over the course of star evolution.

Equations (25), (26) show that, at the accepted values of parameters, the supernova shock moving in the wind of a red supergiant star can accelerate cosmic rays up to somewhat higher energies and the decrease of $p_{\max }$ with time is weaker than in the case of a shock moving in a homogeneous interstellar medium. Also, at a given age of supernova remnant, the value of $p_{\max }$ is essentially higher for the explosion in wind.

\section{Discussion and conclusion}

The generation of turbulence by cosmic-ray streaming instability ahead of the shock is an integral part of cosmic ray acceleration in supernova remnants. We considered this process without limitation on the amplitude of random magnetic field and with allowance for the wave dissipation through Kolmogorov type nonlinearity and by ion-neutral collisions. The amplification of random magnetic field at high shock velocity, $u_{\mathrm{sh}} \gg$ $10^{3} \mathrm{~km} \mathrm{~s}^{-1}$, leads to the increase of maximum energy of accelerated particles above the Bohm limit calculated at the interstellar magnetic field strength. This is in agreement with the results of Bell \& Lucek (2001). On the other hand, in support and development of earlier investigations of Völk et al. (1988), Fedorenko (1990), and Drury et al. (1996) who dealt with not very strong turbulence and considered different nonlinear processes, we found that the nonlinear and linear wave damping may considerably suppress the level of turbulence at $u_{\mathrm{sh}} \lesssim 10^{3} \mathrm{~km} \mathrm{~s}^{-1}$ and thus may decrease the maximum particle energy much below the Bohm limit. On the whole, the maximum energy of accelerated particles with charge $Z e$ rapidly decreases from $\gtrsim 10^{14} \mathrm{Z} \mathrm{eV}$ to much smaller energies $\sim 10^{10} \mathrm{Z} \mathrm{eV}$ during the Sedov stage of supernova remnant evolution at the age from a few hundred years to about $10^{5} \mathrm{yr}$. Our main results are illustrated by Figs. 1 and 2 for the cases of supernova bursts with kinetic energy of ejecta $\mathcal{E}=10^{51} \mathrm{erg}$ in hot rarefied interstellar gas (the gas temperature $T=7 \times 10^{5} \mathrm{~K}$, the average gas density including clouds $n_{0}=10^{-2} \mathrm{~cm}^{-3}$, the density of diffuse gas $n=3 \times 10^{-3} \mathrm{~cm}^{-3}$, the interstellar magnetic field $\left.B_{0}=2 \mu \mathrm{G}\right)$ and in the warm weakly ionized interstellar gas $\left(T=8 \times 10^{3} \mathrm{~K}, n_{0}=0.4 \mathrm{~cm}^{-3}, n=0.1 \mathrm{~cm}^{-3}, B_{0}=5 \mu \mathrm{G}\right) \mathrm{re}-$ spectively. The assumed efficiency of cosmic ray acceleration is $\xi_{\text {cr }}=0.5$.

It is remarkable that the highest energy $E_{\max }$ which the cosmic ray particle can reach in the process of acceleration does not explicitly depend on the value of the interstellar magnetic field, see Eqs. (17), (21). It is easy to check that the highest energy is attained at the time of transition from the free expansion to the Sedov stage of supernova remnant evolution. Using Eq. (19), one can make the following estimate at $t=t_{0}$ (the Sedov solution is not yet applicable at this moment):

$$
\begin{aligned}
E_{\mathrm{max}} & \approx \frac{2 x a C_{\mathrm{cr}}(a) \xi_{\mathrm{cr}} Z m c u_{0}^{2} R_{0}}{V_{\mathrm{a}} r_{\mathrm{g} 0}} \\
& \approx 8 \times 10^{16} Z\left(u_{0} / 3 \times 10^{4} \mathrm{~km} \mathrm{~s}^{-1}\right)^{2} M_{\mathrm{ej}}^{1 / 3} n^{1 / 6} \mathrm{eV}
\end{aligned}
$$

at $\xi_{\text {cr }}=0.5, \varkappa=0.1, a=0.3, n_{0}=n$, the mass of ejecta $M_{\mathrm{ej}}$ is in solar masses. The estimate (27) is in agreement with the estimates made by Bell \& Lucek (2001). The corresponding random magnetic field comprises

$$
\begin{aligned}
\delta B_{\max } & \approx \frac{2 a C_{\mathrm{cr}}(a) \xi_{\mathrm{cr}} u_{0}}{3 V_{\mathrm{a}}} B_{0} \\
& \approx 3 \times 10^{-3} \xi_{\mathrm{cr}}\left(u_{0} / 3 \times 10^{4} \mathrm{~km} \mathrm{~s}^{-1}\right) n^{1 / 2} \mathrm{G}
\end{aligned}
$$

in the upstream region of the size $D\left(p_{\max }\right) / u_{0} \approx \varkappa R_{0} \approx 0.1 R_{0}$. The field can reach more than $\sim 10^{-3} \mathrm{G}$ in the downstream compressed region behind the shock. The value of $E_{\max }$ given by Eq. (27) is by a factor of $\delta B_{\max } / B_{0} \sim 100$ larger than the Bohm value calculated for the interstellar field $B_{0}$.

Equation (25) refers to the case of supernova explosion in the wind of a progenitor star with the characteristic mass loss rate $\dot{M}=10^{-5} M_{\odot} \mathrm{yr}^{-1}$ and the wind velocity $u_{\mathrm{w}}=$ $10^{6} u_{\mathrm{w}, 6} \mathrm{~cm} \mathrm{~s}^{-1}$. 
The estimate of $E_{\max }$ in the case of supernova explosion in the wind of a progenitor star is:

$$
\begin{aligned}
E_{\max } & \approx \frac{2 x a C_{\mathrm{cr}}(a) \xi_{\mathrm{cr}} Z e u_{\mathrm{sh}}^{2} \dot{M}^{1 / 2}}{c u_{\mathrm{w}}^{1 / 2}} \\
& \approx 7 \times 10^{16} Z\left(u_{0} / 3 \times 10^{4} \mathrm{~km} \mathrm{~s}^{-1}\right)^{2} \sqrt{\dot{M}_{-5} / u_{\mathrm{w}, 6}} \mathrm{eV},
\end{aligned}
$$

that in fact coincides with the estimate (27). In both cases the highest particle energy can barely exceed $10{ }^{17} \mathrm{Z} \mathrm{eV}$ even for the extreme value of shock velocity $u_{0}=3 \times 10^{4} \mathrm{~km} \mathrm{~s}^{-1}$.

Two essential assumptions are crucial for our conclusions. The first is the high efficiency of shock acceleration that transfers the fraction $\xi_{\text {cr }} \approx 0.5$ of the upstream momentum flux entering the shock to the cosmic ray pressure and strongly modifies the shock profile. This assumption is based on the results of numerical simulations of diffusive shock acceleration by Berezhko et al. (1996) and Berezhko \& Ellison (1999). The second essential assumption is the high rate of nonlinear Kolmogorov-type dissipation of magnetohydrodynamic turbulence that balance the cosmic ray streaming instability. This assumption is supported by the analytical and numerical investigations of MHD turbulence, see e.g. Goldreich \& Sridhar (1995), Verma et al. (1996), and by the observations of Kolmogorov type spectra of turbulence in the interplanetary (Burlaga 1995) and interstellar (Armstrong et al. 1995) space. It should be pointed out that our description of nonlinear wave interactions is very simplified and can be considered as not more than an order of magnitude estimate. In particular, we ignore the complicated anisotropic structure of magnetohydrodynamic turbulence in $\boldsymbol{k}$-space.

In the case of supernova remnant expansion into partly ionized interstellar gas, the wave dissipation by ion-neutral collisions was also taken into account. Collisional damping may suppresses particle acceleration more than the nonlinear wave dissipation does if the surrounding interstellar gas is not vastly ionized by the supernova burst (the case of type Ia supernova).

The fast decrease of maximum energy of cosmic rays in supernova remnants with time may help to explain why the shell remnants at the age of more than a few thousand years are not prominent sources of $\mathrm{TeV}$ gamma rays produced by very high energy cosmic rays (see e.g. Buckley et al. 1998), in spite of the optimistic preliminary theoretical estimates (Drury et al. 1994; Naito \& Takahara 1994) and in spite of a few detections of gamma-rays from these sources at about $100 \mathrm{MeV}$ (Esposito et al. 1996). On the other hand, a number of young supernova remnants (SN 1006, Cas-A, Tycho, Kepler, RXJ 1713.7-3946) were reported as cosmic ray accelerators of very high energy particles with energies up to $\gtrsim 10 \mathrm{TeV}$ based on the observations of nonthermal synchrotron X-rays or/and $\mathrm{TeV}$ gamma rays. (The effect for the old remnants is evidently compounded by the specific selection of the objects near massive gas clouds that increases the generation of secondary gamma rays through $\pi^{0}$ decays whereas the high gas density $n_{0}$ reduces $p_{\max }$, as is clear from Eqs. (19), (22).) A variety of theoretical constructions by other authors were suggested to resolve this problem (Gaisser et al. 1998; Baring et al. 1999; Kirk et al. 2001; Völk 2001; Malkov et al. 2002) but the ultimate choice will be probably made when the data from the new generation of ground-based (CANGAROO-III, HESS, VERITAS, MAGIC) and space (GLAST) gamma-ray experiments in conjunction with new X-ray satellites will be available.

The accounting for non-linear effects in the instability that accompanies the acceleration of cosmic rays in supernova remnants may simultaneously eliminate two difficulties of modern cosmic ray astrophysics. It raises the maximum energy of accelerated particles in young supernova remnants above the standard Bohm limit through the production of strong random magnetic fields and thus helps to explain the origin of galactic cosmic rays with energies up to $\sim 10{ }^{17} \mathrm{ZeV}$, though it does not cover energies larger than about $3 \times 10^{18} \mathrm{eV}$, even for iron nuclei. This implies an extragalactic origin for cosmic rays with the highest observed energies. It also decreases the maximum energy of particles in the late Sedov stage of supernova remnant evolution, which allows us to explain why these objects are not bright in very high energy gamma rays.

Acknowledgements. This work was stimulated by the discussions with F. Aharonian, E. Berezhko, L. Ksenofontov, and H. Völk during our teamwork on the project "Energetic particles in the Galaxy: acceleration, transport and gamma-ray production" at the International Space Science Institute (Bern). The authors are grateful to Johannes Geiss for sponsorship and kind hospitality at ISSI. This work was supported by RFBR-01-02-17460 grant at IZMIRAN. VSP was also supported by the NASA grant NAG5-11091 during his visit to the University of Maryland where part of this work was carried out.

\section{References}

Achterberg, A. 1981, A\&A, 98, 161

Akhieser, A. I., Akhieser, I. A., Polovin, R. V., Sitenko, A. G., \& Stepanov, K. N. 1975, Plasma Electrodynamics (Oxford: Pergamon Press)

Armstrong, J. W., Rickett, H. J., \& Spangler, S. R. 1995, ApJ, 443, 209

Baring, M. G., Elisson, D. C., Reynolds, S. P., \& Greiner, I. A. 1999, ApJ, 513, 311

Bell, A. R. 1978, MNRAS, 182, 147

Bell, A. R., \& Lucek, S. G. 2001, MNRAS, 321, 433

Bennett, L., \& Ellison, D. C. 1995, JGR, 100, 3439

Berezhko, E. G., \& Ellison, D. C. 1999, ApJ, 526, 385

Berezhko, E. G., Yelshin, V. K., \& Ksenofontov, L. T. 1996, JETP, 82,1

Berezhko, E. G., \& Völk, H. J. 2000, A\&A, 357, 283

Berezinskii, V. S., Bulanov, S. V., Dogiel, V. A., Ginzburg, V. L., \& Ptuskin, V. S. 1990, Astrophysics of Cosmic Rays (Amsterdam: North Holland)

Berezinsky, V. S., \& Ptuskin, V. S. 1989, A\&A, 215, 399

Blandford, R. D., \& Ostriker, J. P. 1980, ApJ, 237, 793

Boulares, A., \& Cox, D. P. 1988, ApJ, 333, 198

Buckley, J. H., Akerlof, C. W., Carter-Lewis, D. A., et al. 1998, A\&A, 329,639

Burlaga, L. 1995, Interplanetary magnetohydrodynamics (New York: Oxford Univ. Press)

Bykov, A. M., Chevalier, R. A., Ellison, D. C., \& Uvarov, Y. A. 2000, ApJ, 538, 203

Chin, Y., \& Wentzel, D. G. 1972, Ap\&SS, 16, 465

Cho, J., \& Vishniac, E. T. 2000, ApJ, 539, 273

Drury, L. O'C., Ellison, D. E., Aharonian, F. A., et al. 2001, Space Sci. Rev., 99, 329

Drury, L. O’C., Aharonian, F. A., \& Völk, H. J. 1994, A\&A, 287, 959 
Drury, L. O’C., Duffy, P., \& Kirk, J. G. 1996, ApJ, 309, 1002

Esposito, J., Hunter, S. D., Kanbach, G., \& Sreekumar, P. 1996, ApJ, 461,820

Falle, S. A. E. G., \& Giddings, J. R. 1987, MNRAS, 225, 399

Fedorenko, V. N. 1990, Preprint 1442, A. F. Ioffe Phys. Tech. Inst., Leningrad

Fedorenko, V. N., Ostryakov, V. M., Polyudov, A. N., \& Shapiro, V. D. 1990, Sov. J. Plasma Phys., 16, 443

Gaisser, T. K., Protheroe, R. J., \& Stanev, T. 1998, ApJ, 942, 219

Goldreich, P., \& Sridhar, S. 1995, ApJ, 438, 763

Goldstein, M. L., Roberts, D. A., \& Matthaeus, W. H. 1995, ARA\&A, 33,283

Hu, Y. Q., Habbal, S. R., \& Li, X. 1999, JGR, 104, 24819

Jones, F. C., Lukasiak, A., Ptuskin, V. S., \& Webber, W. R. 2001, ApJ, 547,264

Kirk, J. G., \& Dendy, R. O. 2001, J. Phys. G, 27, 1589

Kulsrud, R. M. 1978, Phys. Scr., 2(1), 177

Kulsrud, R. M., \& Cesarsky, C. J. 1971, Astro. Lett., 8, 189

Lagage, P. O., \& Cesarsky, C. J. 1983, A\&A, 118, 223

Lee, M. A., \& Völk, H. J. 1973, Ap\&SS, 24, 31

Livshits, M. A., \& Tsytovich, V. N. 1970, Nucl. Fusion, 10, 241
Lozinskaya, T. A. 1992, Supernovae and Stellar Wind in the Interstellar Medium (New York: American Institute of Physics) Lucek, S. G., \& Bell, A. R. 2000, MNRAS, 314, 65 Malkov, M. A., Diamond, P. H., \& Jones, T. W. 2002, ApJ, 571, 856

Malkov, M. A., \& Drury, L. O'C. 2001, Rep. Prog. Phys., 64, 429

McKenzie, J. F., \& Völk, H. J. 1982, A\&A, 116, 191

Naito, T., \& Takahara, F. 1994, J. Phys G, 20, 477

Ostriker, J. P., \& McKee, C. F. 1998, Rev. Mod. Phys., 60, 1

Parker, E. N. 1958, ApJ, 128, 664

Reynolds, S. P. 1998, ApJ, 493, 375

Saur, J., \& Bieber, J. W. 1999, JGR, 104, 9975

Seaton, M. J. 1959, MNRAS, 119, 81

Skilling, J. 1975, MNRAS, 173, 255

Verma, M. K., Roberts, D. A., Goldstein, M. L., Ghosh, S., \& Stribling, W. T. 1996, JGR, 101, 21619

Völk, H. J. 2001, Proc. XXXVIth Renc. de Moriond, Les Arcs, France [astro-ph/0105356]

Völk, H. J., \& Biermann, P. L. 1988, ApJ, 333, L65

Völk, H. J., Drury, L. O’C., \& McKenzie, J. F. 1984, A\&A, 130, 19

Völk, H. J., Zank, L. A., \& Zank, G. P. 1988, A\&A, 198, 274

Zirakashvili, V. N. 2000, JETP, 114, 810 